\title{
A paradox of social distancing for SARS-CoV-2: loneliness and heightened immunological risk
}

\author{
Liron Rozenkrantz $\mathbb{1}^{1} \cdot$ Michael H. Bernstein ${ }^{2} \cdot$ Christopher C. Hemond ${ }^{3}$
}

Received: 9 June 2020 / Revised: 27 July 2020 / Accepted: 29 July 2020 / Published online: 10 August 2020

(c) Springer Nature Limited 2020

\section{"Of all the diseases that I have known, loneliness is the worst"-Mother Theresa}

The World Health Organization declared the SARSCoV-2 virus a global pandemic in March of 2020. In an effort to reduce the harms and rate of exponential spread, regional and national governments across the world instituted a variety of measures. These have included orders for citizens to practice social distancing, which in the US has affected over 300 million people. In their most extreme, these social distancing measures are isolation orders to "shelter in place", at one point affecting $\sim 17$ million Americans. Data regarding the effects of these policies are emerging, but two outcomes include greater social isolation and likely increased loneliness. An important distinction arises between these two concepts. Social isolation is the objective lack of, or reduction in, social contact. Loneliness is the subjective discrepancy between the desired and actual levels of social connection [1]. Objective social isolation and subjective loneliness are only weakly correlated $(r \sim$ 0.2 ), but both have independent real-world health consequences and are associated with long-term increases in mortality ( $29 \%$ and $26 \%$, respectively). The magnitude of

These authors contributed equally: Liron Rozenkrantz, Michael $\mathrm{H}$ Bernstein, Christopher C. Hemond

Liron Rozenkrantz

lironroz@mit.edu

1 Massachusetts Institute of Technology, McGovern Institute for Brain Research, Cambridge, MA 02139, USA

2 Department of Behavioral and Social Sciences, Center for Alcohol \& Addiction Studies, Brown University, Providence, RI, USA

3 Department of Neurology, University of Massachusetts, Amherst, MA, USA these effects rival that of smoking and obesity on long-term health risks [1]. Emerging evidence for the social repercussions of the pandemic is worrisome; a recent longitudinal study following more than 35,000 people reported that while overall loneliness has not changed during the COVID pandemic, individuals who described high levels of baseline social isolation are now experiencing significantly worse pandemic-related loneliness [2]. Now more than ever the most socially vulnerable would likely benefit from clinical assessment and support. Our own unpublished survey data $(N=155)$ indicate that $60 \%$ of respondents from an online campaign in the USA, Israel, and UK report a greater sense of loneliness since the pandemic began.

Feelings of loneliness are the brain's emotional response to perceptions of isolation, and serve to motivate individuals to seek social connections. As highly social mammals, humans depend on interpersonal connections for mutual health and protection. In times of lack, the neurobiological correlates of social craving are similar to that of hunger. A recent neuroimaging study found that the same valuation regions which are activated at the sight of food cues following a day of fasting are activated at the sight of social cues after a day of social isolation, highlighting the human need of socialization [3]. Along these lines, prolonged solitary confinement has been recognized as a form of torture by the United Nations. A powerful psychological stress is felt in the absence of social protection or assistance, which, via the neuroendocrine and sympathetic nervous systems, activates a conserved immunological program optimized for self-preservation. This immunological program is shifted towards evolutionary threats characteristic of social isolation (e.g., violence, predation, and trauma), while fewer resources are afforded to fight internal threats such as (viral) infectious transmission and neoplasms.

Specifically, loneliness is associated with decreased expression of interferon response factors and antibody synthesis necessary for antiviral defense. A landmark 
1991 study demonstrated that baseline stress levels predicted a dose-dependent response to coronavirus type 229E; higher stress was independently associated with greater risk of infection and symptom severity [4]. In a follow-up, Cohen et al. [5] infected volunteers with an upper respiratory virus (rhinovirus). Participants with fewer social ties were more susceptible to infection, showed increased viral shedding, and developed more severe symptoms. An additional study administered influenza vaccinations to 83 college freshmen and found increased levels of loneliness to be associated with poorer antibody response, mediated by greater psychological stress [6]. These studies, among others in the Common Cold Project (https://www.cmu.edu/common-cold-project), provide strong evidence that higher levels of social engagement represent a protective effect against the inflammatory immune response to respiratory viruses. This is independent of other lifestyle and demographic factors.

Loneliness additionally predisposes the innate immune response toward proinflammatory gene expression, increasing the number of circulating monocytes and upregulating expression of proinflammatory $\mathrm{NF}-\mathrm{Kb}$ and AP-1 proteins [7]. This adaptation is effective in the short term to prepare for threats of bodily harm, bacterial infection, or trauma. However, chronic activation of this immune response is associated with a multitude of inflammatorymediated illnesses including increased incidence of neoplastic, cardiovascular, and neurodegenerative diseases [7]. We speculate that this proinflammatory predisposition may also be a risk for the development of the highly lethal "acute respiratory distress syndrome" responsible for significant mortality in COVID-19. Although unlikely to manifest as a substantial individual consequence of short-term social distancing, these covert threats are important to consider in decisions surrounding social distancing measures, because a small effect distributed over a large population can have public health consequences.

The evidence summarized here indicate a catch-22. On the one hand, social distancing is needed to protect people from spreading SARS-CoV-2. On the other hand, these distancing measures may ironically be exacerbating the widespread loneliness already reported by seniors, while simultaneously undermining the exact immunological resources needed to appropriately respond to potential SARS-CoV-2 infection [8]. One conclusion from these data is the need for targeted countermeasures. This can begin with words: replacing the term "social distancing" with "physical distancing", as endorsed by the WHO, will explicitly emphasize that the weakening of social connections is neither a goal nor a policy target. Although uncertainty, stress and anxiety are inherent to a global pandemic, loneliness need not be a foregone conclusion.
Evidence-based interventions to reduce loneliness remain sparse, but any form of positive social support is likely to buffer or reverse perceived isolation. Many people have naturally increased their use of technology (e.g., videoconferencing software) to remain socially engaged with colleagues, acquaintances, and loved ones. While the psychological advantages of this are self-evident, it may also confer immunological benefits through the mechanisms described in this paper. The healthcare system itself could be better adapted to reaching those most in need, providing assessments of objective and subjective social isolation for at-risk individuals and connecting them with community-based resources. Lastly, although more research is needed to explore pharmacological and effective technology-based behavioral interventions targeting subjective social isolation, several mind-body interventions such as yoga, (mindfulness) meditation, and tai chi have demonstrated early promise in reversing the harmful inflammatory biological effects of adverse social circumstances [7, 9]. COVID-19 appears to be inspiring the proliferation of freely accessible online classes and activities like yoga and mindfulness as well. While social isolation is associated with paradoxical immunological risks in this pandemic, lifestyle modifications may attenuate these changes and have important public health implications for the coming months in the ongoing fight against SARSCoV-2.

\section{Compliance with ethical standards}

Conflict of interest The authors declare that they have no conflict of interest.

Publisher's note Springer Nature remains neutral with regard to jurisdictional claims in published maps and institutional affiliations.

\section{References}

1. Holt-Lunstad J, Smith TB, Baker M, Harris T, Stephenson D. Loneliness and social isolation as risk factors for mortality: a metaanalytic review. Perspect Psychol Sci. 2015;10:227-37.

2. Bu F, Steptoe A, Fancourt D. Loneliness during lockdown: trajectories and predictors during the COVID-19 pandemic in 35,712 adults in the UK. https://www.medrxiv.org/content/10.1101/2020. 05.29.20116657v1.

3. Tomova L, Wang K, Thompson T, Matthews G, Takahashi A, Tye $\mathrm{K}$, et al. The need to connect: acute social isolation causes neural craving responses similar to hunger. https://www.biorxiv.org/ content/10.1101/2020.03.25.006643v1.

4. Cohen S, Tyrrell DAJ, Smith AP. Psychological stress and susceptibility to the common cold. N Engl J Med. 1991;325:606-12.

5. Cohen S, Doyle WJ, Skoner DP, Rabin BS, Gwaltney JM. Social ties and susceptibility to the common cold. JAMA. 1997;277:1940-4. 
6. Pressman SD, Cohen S, Miller GE, Barkin A, Rabin BS, Treanor JJ. Loneliness, social network size, and immune response to influenza vaccination in college freshmen. Health Psychol. 2005;24:297-306.

7. Cole SW. The conserved transcriptional response to adversity. Curr Opin Behav Sci. 2019;28:31-7.
8. Taylor HO. Social isolation's influence on loneliness among older adults. Clin Soc Work J. 2020;48:140-51.

9. Bower JE, Irwin MR. Mind-body therapies and control of inflammatory biology: a descriptive review. Brain Behav Immun. 2016;51:1-11. 